\title{
Versos y tratados en la Ilustración científica (1650-1750)
}

\author{
Carlos M. Collantes Sánchez \\ Universidad de Córdoba
}

CES.XVIII, núm. 25 (2015), págs. 75-96. 


\section{RESUMEN}

Estudiamos la poesía impresa en los libros de ciencia publicados en España entre 1650 y 1750. En esta época del Bajo Barroco, la ciencia española comienza a despegar gracias, en parte, a la figura de los novatores y sus obras. Hemos analizado cuantitativa y cualitativamente los versos, tanto paratextuales como intratextuales, que los novatores e impresores tenían a bien plasmar en las obras. El objetivo del trabajo es conocer la función y finalidad de dichas poesías, y ver si estos versos responden a las prácticas editoriales que se asentaron en la centuria anterior. Buscamos comprender la relación que une a los tratados científicos y sus poesías.

Palabras clave

Ilustración, Bajo Barroco, Ciencia, Poesía, Imprenta.

\section{AbSTRAC}

We study the poetry printed in the books of science published in Spain between 1650 and 1750. In this time of the Bajo Barroco, the Spanish science begins to grow to the figure of the novatores and his works. We have analyzed quantitatively and qualitatively these verses, so many paratextuales as intratextuales, that the novatores and the printers have printed in his books. The aim of the work is to know the function and purpose of the above mentioned poetry, and to observe if these verses answer to the publishing practices that settled themselves in the previous century. We seek to understand the relation that joins to the scientific agreements and his poetry.

KEYWORDS

Illustration, science, poetry, press, Late Baroque.

Recibido: 10 de junio de 2015. Aceptado: 3 de septiembre de 2015. 
En un artículo publicado hace casi veinte años François Lopez ${ }^{1}$ sostenía que el período que sigue al Barroco se encontraba insuficientemente explorado. No sería apropiado decir que nos encontramos en la misma situación, ya que en los últimos tiempos ha aumentado tanto la bibliografía como también los coloquios y congresos al respecto. Lopez comentaba la falta de consenso incluso para la denominación del período. Ante las propuestas de Pre-Ilustración o Ilustración Temprana, el autor proponía que dicho periodo, que fijaba entre 16701750, era demasiado extenso y con disparidades como para aunarlo bajo un solo marbete; de este modo propuso la distinción cronológica del 1670 al 1726 como «tiempo de los novatores» y del 1726 al 1760 como la «época de Feijoo, donde predominó la divulgación de la lengua española y las ciencias modernas». Sin entrar a valorar dichas denominaciones ${ }^{2}$ me interesa mucho cómo Lopez centraba en los novatores y la ciencia los pilares para la separación temporal ${ }^{3}$.

Nuestra indagación va encaminada por esos lares. En las fechas comentadas los países europeos se mostraban muy dispuestos a participar de forma conjunta en una nueva visión europea, que despegaba sobre las alas de la ciencia y sus avances, mientras que España, tal vez por los últimos estertores contrarreformistas, quedaba a la zaga. La fuerte presión ejercida por la Iglesia sobre la sociedad y los propios avances científicos creaba una película impermeable que impedía que esta nueva visión calase, aunque no del todo. Lopez afirmaba que esa impermeabilidad no podría ser absoluta, basándose en los datos de producción, comercio y circulación del libro desde las Provincias Unidas a España ${ }^{4}$. No solo tardaban en arraigar dichas ideas, sino que conseguían una clara y manifiesta oposición a ellas. Lo que queda patente es que este (lento) avance procedía de las ciencias, tanto las puras como las aplicadas.

1 François Lopez, «Los novatores en la Europa de los sabios», en Studia historica. Historia moderna, 14 (1997), págs. 95-111.

2 Más teniendo una formula propuesta y a mi parecer idónea, como «Bajo Barroco» expuesta por Pedro Ruiz Pérez, «Para la historia y la crítica de un período oscuro: la poesía del Bajo Barroco», en Calíope. Journal of the Society for Renaissance and Baroque Hispanic Poetry, 18.1 (2012), págs. 9-25.

3 Una de las mejores obras que contextualiza y explica el movimiento novator es Jesús PérEz MAGALLón, Construyendo la modernidad: La cultura española en el tiempo de los novatores (1675-1725), Madrid, CSIC, 2002.

4 Lopez, «Los novatores en la Europa de los sabios», pág. 108. 
Por este motivo, ahora que los estudios sobre la poesía bajobarroca van aumentando, creo que sería interesante que nos fijásemos en aquellos versos que aparecían impresos en las obras científicas castellanas, ya fuesen en preliminares o en las entrañas de la propia obra. Me estoy refiriendo a poesías completas, autónomas del texto principal. Si podemos considerar a esos libros científicos como el devenir, o como oposición a ese futuro que se vislumbraba en Europa, ¿qué papel tenía la poesía? ¿El fin poético se correspondía con la naturaleza de la obra?; y si fuese así, ¿qué tipo de poesía era la utilizada y por quién? Espero que a la conclusión de esta breve cala pueda dar respuesta a estas preguntas como conclusiones, no definitorias, pero sí aproximativas.

Para responder a estas cuestiones necesitábamos un corpus amplio, fiable y representativo del período. Optamos por los fondos de la Biblioteca Digital Hispánica $^{5}$, aun a sabiendas de las limitaciones y problemáticas que acarreaba, que no eran pocas. El mayor inconveniente es que los datos no tienen una exactitud completa, al no ser todavía exhaustivo, por lo aleatorio de la decisión de qué materiales son los que se deben digitalizar por parte de la biblioteca. Asimismo es revisable la categorización de las obras dentro de la clasificación de materias. Aun así, el corpus obtenido es lo suficientemente aproximativo como para ensayar unas calas de cierta validez sintomática ${ }^{6}$.

Los registros que conforman nuestro corpus se agrupan en las colecciones de Ciencias puras y naturales ${ }^{7}$ y Ciencias aplicadas ${ }^{8}$. En esta aproximación positivista y cuantitativa hemos obtenido los siguientes resultados.

5 La Biblioteca Digital Hispánica (desde ahora BDH), tal y como reza en su web (http://www.bne.es/ es/Catalogos/BibliotecaDigitalHispanica/Inicio/) es el «portal libre y gratuito de documentos digitalizados de la Biblioteca [Nacional]». Fue creada en 2008 y entre sus fondos encontramos «libros impresos entre los siglos XV y XIX, manuscritos, dibujos, grabados, folletos, carteles, fotografías, mapas, atlas, partituras, prensa histórica y grabaciones sonoras».

6 El corpus utilizado para el trabajo no es exhaustivo pero lo que nos interesa de él es su carácter de muestreo con valor representativo y las conclusiones (provisionales) que podemos obtener. Por tanto, la metodología desarrollada se encamina al interés por conocer la función de los versos y las diferentes estrategias de uso de los mismos, así como sus opciones métrico-formales.

7 La BDH agrupa dentro de las Ciencias puras y Ciencias naturales las siguientes disciplinas: Naturaleza y Medio ambiente, Matemáticas, Astronomía y Astrofísica, Física, Química y Ciencias minerales, Ciencias geológicas, Paleontología y Fósiles, Ciencia biológicas, Botánica y Zoología.

8 En cambio, dentro del marbete de Ciencias aplicadas encontramos: Ciencias médicas, Ingeniería y Tecnología, Agricultura, Economía doméstica, Industria y Comercio, Tecnología química, Industrias y Oficios diversos, Industrias de artículos acabados e Industrias de la construcción. Evidentemente, subcategorías tanto de Ciencias puras y naturales como aplicadas quedan totalmente fuera cuando hablamos de libros impresos comprendidos entre 1650-1750. Es necesario realizar una búsqueda aplicado los filtros de: tipo de material libro, filtro por años 1650-1750 y filtro por lengua Español. 
Para la colección de Ciencias puras, de un total de 3.017 obras digitalizadas, tenemos 52 obras que se amoldan a nuestros criterios. Hemos estudiado cada una de las obras, y en 24 de ellas hay composiciones poéticas autónomas. La mayoría de estos versos son paratextuales y se encuentran en los preliminares de las obras, aunque no todos.

Muchas de estas obras con poesía son títulos importantes en el discurrir científico del momento, con autores considerados dentro del movimiento de los novatores. Me detendré en las más destacadas.

La obra Compendio mathematico: en que se contienen todas las materias mas principales de las ciencias de Tomás Vicente Tosca ${ }^{9}$ fue muy influyente en su área científica. El clérigo de la Congregación del Oratorio de San Felipe Neri formó parte del núcleo de los novatores valencianos, que según Vicenç Roselló «tuvo como principales mentores a Félix Falcó y Baltasar Iñigo, el primero de los cuales actuó de enlace con... Tosca y Joan Baptista Coratjá (o Corachán)» ${ }^{10}$. La obra estuvo inspirada «básicamente en el Cursus seu mundus mathematicus (1690) del jesuita francés Claude François Milliet Dechales» ${ }^{11}$, editada por primera vez en 1707-1715 y reeditada póstumamente en 1727 y 1757 . Tosca dedica la primera edición de 1707 al rey Felipe V, el animoso, mientras que la segunda edición, póstuma (1727), la dedica Jaime Marqués y López al conde de Aranda, cuando aún vivía y reinaba el citado rey y había muerto Tosca. Estos datos son reveladores para nuestra investigación, ya que en la primera edición no había ninguna poesía paratextual en la obra, mientras que en la segunda encontramos una composición en octavas, de 200 versos y con 22 anotaciones, firmada por el mismo Jaime Marqués y dedicada a Buenaventura Pedro Alcántara Jiménez de Urrea, conde de Aranda, sustituyéndose además una imagen del rey de la primera edición por el escudo de armas del noble en la segunda. Tosca buscó para su obra la mayor protección posible en la figura monárquica, pero, muerto el clérigo de San Felipe Neri, Jaime Marqués adquirió en 1723 el privilegio de impresión de la obra por diez años y cambió el dedicatario de la misma para obtener el mecenazgo de un Grande de España. Para el editor de la segunda edición,

9 Tomás Vicente Tosca, Compendio mathematico en que se contienen todas las materias mas principales de las ciencias que tratan de la cantidad, Valencia, por Antonio Bordazar, 1707-1715. Segunda impression corregida y enmendada... Madrid, en la imprenta de Antonio Marin: se hallarà en la librería de Juan de Moya ... y en casa de D. Jayme Marquès ..., 1727.

10 Vicenç M. Rosselló, «Tomàs V. Tosca y su entorno ilustrado en Valencia. Obra autógrafa y atribuciones», en Ería, 64-65 (2004), pág. 160.

11 Rosselló, «Tomàs V. Tosca y su entorno ilustrado en Valencia», pág. 160. 
afincado en la corte, era más provechoso el amparo del conde de Aranda que el utilizar la misma dedicatoria del fenecido valenciano. La composición poética es un ensalzamiento y enlazamiento de la familia del conde de Aranda con la flor y nata de las familias nobles españolas y valencianas. Más allá de la propia composición, nos interesa que a través de ella se dedica una obra consagrada de un novator a un noble (en detrimento de la figura regia, con su valor simbólico) como una estrategia editorial, no solo por los elementos mencionados, sino también por la inversión económica que suponen, por una parte, las correcciones de «los muchos errores cometidos en la primera impresión, originados, como se debe creer, de mal cuidada la estampa, y del manifiesto descuido de quien talló las láminas» ${ }^{12}$, y por otra, la impresión de un pliego más (en el que se contiene el poema-dedicatoria) inexistente en la primera aventura editorial de la obra.

En tres obras de otro conocido científico valenciano, Jerónimo Cortés ${ }^{13}$, hallamos diversas composiciones poéticas. Nos centramos en la obra Tratado de los animales terrestres, y volatiles, $y$ sus propriedades ${ }^{14}$, reeditada por tercera vez en 1672. De este temprano título de divulgación científica afirma Francisco Javier Peris Felipo «que cabe destacar su interés por incidir en la zoología y su indudable valor como contribución valenciana a la historia de la ilustración biológica de los primeros años del siglo XVII» ${ }^{15}$. El autor tuvo una producción científica considerable, y en la mayoría de sus obras podemos encontrar composiciones poéticas. En la obra seleccionada hallamos un romance, dos octavas reales y una quintilla, composiciones completas no paratextuales que forman parte del discurso. Estas composiciones tienen una clara finalidad de ayudar al lector en la compresión y memorización del texto, ya que son versos populares traídos a colación de la temática de la obra; tal sucede con la loa y privilegios del Asno o los refranes del Gallo. Toda la obra contiene versos de tradición popular, y de esta forma se transmitieron los conocimientos de la biología pre-ilustrada del último tercio del siglo XVII. En su artículo, Peris Felipo anota cómo, en relación a la figura del autor, «López Piñero, Navarro Brotons y Portela Marco lo consideran como uno

12 Tosca, Compendio mathematico, pág. [26].

13 El científico murió en el primer tercio del siglo XVII pero sus obras se siguieron reeditaron a lo largo de esa centuria. Consideramos por tanto que sus obras cobran un doble valor: son antecedentes directos a los movimientos novatores y también, por sus reediciones, títulos hábiles de consulta y apoyo en el comienzo del despliegue científico español. Así, las reediciones de sus trabajos impresos a partir de 1650 tienen validez para nuestra propuesta.

14 Jerónimo CoRTÉs, Tratado de los animales terrestres, y volatiles, y sus propriedades, compuesto por..., Valencia, Imprenta de Benito Macè a costa de Francisco Duart, 1672. (Como se indica en el catálogo de la Biblioteca Nacional, hay al menos dos ediciones de este mismo año).

15 Francisco Javier Peris, «Aportación a la divulgación zoológica valenciana del siglo XVII. El Tratado de los animales terrestres y volátiles de Jerónimo Cortés (1613)», Revista Historia Autónoma, 2 (2013), págs. 59-74. 
de los autores representativos de la "subcultura científica extraacadémica" ${ }^{16}$. Este modelo de divulgación de las ciencias naturales estaba compuesto por texto (científico) e ilustraciones, pero en este caso conserva aún reminiscencias de tradición oral plasmadas en versos, que apoyan la teoría científica. En la obra se percibe la influencia de Plinio entre otras autoridades clásicas como Aristóteles, Ovidio o Dioscórides y algunas bíblicas, principalmente, Job e Isaías.

Y comparado con Plinio y encumbrado a su altura se nos presenta a Juan Bautista Juanini en el soneto paratextual de su obra Nueva idea physica natural demonstratiua..${ }^{17}$, escrito por el franciscano fray José Antonio de Hebrera y Esmir. Doctor de origen italiano, a Juanini se le reconoce como uno de los principales actores del movimiento novator ${ }^{18}$. Más interesa la figura del poeta aragonés, religioso y hombre de letras, que escribió el Jardín de la elocuencia ${ }^{19}$, obra centrada en retórica y elocución. Monge nos dice de la obra que «La "ortodoxia" es total en lo que toca a la tradición retorica. Ni es original ni pretende serlo. Sus "autoridades" son las de siempre: Aristóteles, Cicerón, Séneca, Quintiliano. Y ello encaja con el aludido carácter escolar que Hebrera atribuye a su "Iardin" (y con lo que es ya dicho común: la falta de originalidad de las Retóricas españolas a partir de principios del siglo XVII) ${ }^{20}$ pero son los poetas del Barroco los usados para los ejemplos de la obra (con finalidad educativa): «Los más representados son, por este orden, Góngora (once veces), Lope (ocho), Juan Pérez de Montalbán (siete) y Luis de Ulloa y Pereira (seis) ${ }^{21}$. Con esto quiero decir, sin considerarlo una certeza y menos sin haber podido estudiar la obra del aragonés a fondo, que en lo tocante a su poesía, esta se muestra más conservadora si se compara con las obras de los novatores coetáneos. Pero llama la atención cómo es este religioso quién preludia en verso a un autor novator. Aunque por otro lado, nos comenta en su artículo Monge, el aragonés «sigue el postulado de los enemigos de la moda

\footnotetext{
16 Peris, «Aportación a la divulgación zoológica valenciana del siglo XVII», pág. 61.

17 Juan Bautista JuANini, Nueva idea physica natural demonstratiua, origen de las materias que mueuen las cosas, compuestas de la porcion mas pura de los elementos... formacion del firmamento, y causas segundas de los sublunares parte primera... escriuela —, Zaragoza, Por los herederos de Domingo la Puyada, 1685.

18 Remito para conocer su vida y obra a la tesis doctoral de Jesús V. Coвo Gómez, Juan Bautista Juanini (1632-1691). Saberes médicos y prácticas quirúrgicas en la primera generación del movimiento novator, Barcelona, Universitat Autònoma de Barcelona, 2005.

19 José Antonio de Hebrera y Esmir, Jardín de la elocuencia, edición y prólogo de Félix Monge, Zaragoza, Universidad de Zaragoza, 1959. Esta obra no forma parte del corpus analizado pero su mención es relevante ya que a través de ella podemos conocer el pensamiento y posicionamiento de Hebrera, autor del poema paratextual de la citada obra de Juanini.

20 Félix Monge, «Una retórica aragonesa de fines del siglo XVII», en VV. AA., Gracián y su época : actas de la I Reunión de Filólogos Aragoneses. Ponencias y comunicaciones, Zaragoza, Institución Fernando el Católico, 1986, pág. 105.

${ }^{21}$ Monge, «Una retórica aragonesa de fines del siglo XVII», pág. 110.
} 
culta» ${ }^{22}$, proclamando en cambio la claridad como valor y defendiendo el uso de palabras castizas, proprias y significativas; también previene contra extranjerismos y contra el insufrible estilo inchado. Son las mismas consignas que arraigarán (ya lo estaban haciendo) en las mentalidades literarias más ilustradas, ahora sí más acordes con la obra que imprime su propia poesía paratextual.

No podemos detenernos en todos los títulos, pero sí destacamos a los autores de algunos de ellos: Aznar de Polanco, Pedro Manuel Cedillo o Antonio Fuentelapeña, y a hombres de letras como Torres Villarroel o Francisco Botello de Moraes. Todos estos letrados ${ }^{23}$ fueron personalidades relevantes en el panorama social español del momento y los versos contenidos en sus obras merecerían mayor detenimiento en un futuro trabajo.

Como última cata cualitativa dentro de las ciencias puras estudiaremos con detenimiento poesías científicas que también forman parte del corpus analizado. En la obra Causas eficientes y accidentales del fluxo y refluxo del mar y de sus notables diferencias con la diuersidad de corrientes... del orbe aquati $2^{24}$ del Capitán Pedro de Castro aparecen cuatro sonetos paratextuales con un valor destacado ${ }^{25}$. El propio texto señala que los poemas aparecen «por principio; pues son quatro fundamentos deste breve Tratado»; de esta forma el propio autor anticipa el contenido de la obra, a mi parecer, dándole un plus de calidad literaria al texto científico, tal vez con un objetivo divulgador y comercial, como el propio autor indica: «Por la novedad, presumo emplearás tu dinero en este Libro: apréciale como esclavo, por sus yerros; que aunque sea el peor, si quieres enajenarle, a ley de hombre de verdad, con los defectos que tuviere, has de manifestar lo menos malo en que sirve». Además, la idea toma más consistencia si tenemos en cuenta que la obra pretende (práctica común en la época) enmendar la plana a otra de la misma materia, de Francisco de Seyxas (Theatro naval...). Merece la pena destacar que el vocabulario empleado en los poemas señalados es muy especializado, con términos como: primer móvil, orbe, fluxos, globo, universo..., o cenit y nadir, trepidación. Estos mismos términos los hallamos en otras muchas composiciones líricas que forman parte del corpus. Se aprecia cómo este vocabulario torna la sensibilidad poética de las composiciones estudiadas hacia un aspecto más explicativo. Estas no buscan apelar a la sensibilidad del lector,

22 Monge, «Una retórica aragonesa de fines del siglo XVII», pág. 111.

23 Más específicamente «literatos».

24 Pedro de Castro, Causas eficientes y accidentales del fluxo y refluxo del mar y de sus notables diferencias con la diuersidad de corrientes... del orbe aquatil, Madrid, En la imprenta de Manuel Ruiz de Murga, 1694.

25 Estos sonetos forman parte del paratexto «Prólogo al Lector» y tienen la función de contextualizar al lector con los contenidos que encontrará en el tratado. Los sonetos llevan por título: «Refluxo del primero movil», «Fluxo que ocasiona la luna», «Fluxo del movimiento de trepidacion» y «Refluxo de lo que el sol supura». Castro, Causas eficientes y accidentales del fluxo y refluxo del mar, págs. [22-24]. 
sino reivindicar unas características afines con la profesión y ámbitos de sus compositores, que como veremos, no son poetas profesionales.

Mayor detenimiento merece el romance endecasílabo de Álvaro Cortés Aranda y Villalón, filósofo y teólogo del Ilustre Colegio de San Pablo de Córdoba, que se imprimió en la obra Tablas philipicas catholicas o generales de los movimientos celestes ${ }^{26}$, de Juan Bautista Riccioli, traducida, ampliada, editada e impresa por Gonzalo Antonio Serrano. Este último fue médico y astrónomo de la ciudad de Córdoba, donde tenía su propio observatorio e imprenta, de la cual salieron abundantes obras científicas.

El poema de Álvaro Cortes, impreso en los preliminares de la obra junto con otras composiciones poéticas, tiene una extensión de 452 versos y como título Principio, origen y progresos de la alta y verdadera ciencia astronómica. El marbete en sí ya es revelador, sobre todo si lo encuadramos dentro de una obra importante de la ciencia y en la fecha de mediados del siglo XVIII, pues evidencia cómo la narración histórico-cronológica de la ciencia que nos muestra es la verdadera ${ }^{27}$. Aunque el poema no puede ser analizado como un axioma del devenir de la historia de la ciencia, sí puede leerse como un acercamiento bastante razonable a la mentalidad científica del momento, aún convulsa entre diferentes factores (muchas veces contrapuestos), como la religión, la fe, la ciencia y los avances ilustrados. No podemos olvidar que aún la Inquisición tenía un papel en el control ideológico y divulgativo de los saberes.

En el poema encontramos una nómina de «científicos» que se remonta al primero de los hombres, Adán, y termina con el propio Gonzalo Antonio Serrano. Esta conjunción de personalidades bíblicas junto con científicos constatados es la visión de una ciencia aún constreñida por la religión. Entre los nombres bíblicos encontramos a Adán, Seth, Noé, San Lucas y Salomón. El resto del canon está formado por pensadores antiguos (Ptolomeo, Tiburtino, Averroes, Séneca, Avicena, Lucano, Claudio, Alphonso Rey, Theón, Proclo, Theodosio, Menelao, Jeronio, Julio Firmico, Sereno, Vitelion...) y científicos europeos modernos (Beyerlink, Dechales, Bulialdo, Juan el Magno, Purbachio, Leopoldo el Austriaco, Copérnico, Reinholdo, Erasmo, Tycho Brahe, Keplero, Juan Antonio Magino, Lansbergio, Argoli, Ricciolo...), finalizando la nómina y cerrando el poema con el traductor y editor de la obra, Gonzalo Antonio Serrano.

Parece muy interesante este canon de personalidades, ya que en otra obra impresa anterior, también por Gonzalo Antonio Serrano (Astronomía universal,

26 Giovanni Battista Riccioli, Tablas philipicas catholicas o generales de los movimientos celestes... traducidas de idioma latino al castellano, corregidas, y aumentadas... por el Doctor D. Gonzalo Antonio Serrano... Tomo segundo, Córdoba, en la imprenta del author..., por Antonio Serrano, 1744.

${ }^{27}$ Al menos para los científicos coetáneos. 
teórica y práctica ${ }^{28}$ ), encontramos en el prólogo (unas 74 páginas en tamaño folio) una guía bio-bibliográfica con los nombres de los científicos y los avances que realizaron en la ciencia astronómica, titulada Progresso de la Astronomía, con orden Alfabetico de sus Autores, y tiempos en que florecieron. El autor del romance heroico era discípulo de Gonzalo Antonio Serrano, de manera que podemos afirmar que se pudo basar en esta obra para la realización de su canon poético-científico (al igual que en la propia sapiencia de su maestro inculcada durante las clases), de ahí que el título del poema impreso diez años más tarde sea muy similar al de la guía. El cotejo de esta bio-bibliografía, la obra citada en su conjunto y el romance heroico, corrobora esa mentalidad científica confusa del momento español por los siguientes motivos: 1) la ausencia, en el poema, de Galileo, no así en la guía, en que se dice que es «excelente astrónomo», y se continúa señalando: «Aunque los modos, con que explicó Galileo el Sistema Copernicano, le ocasionaron la censura de la Sacra Inquisición de Roma; no obstante, fue conocido por uno de los mayores ingenios, que han ilustrado la Astronomía» ${ }^{29}$; 2 la mención en el poema de Copérnico, pasando casi de puntillas por su teoría heliocéntrica, como mostramos:

Nació luego el ilustre torinense

Copérnico, motor de aquel gallardo

sistema excogitado antiguamente

por el samio Aristarco y Philolao,

aquel a quien el nombre se adjudica

de hipótesis supuesto imaginario,

pues cual centro supone a el sol inmóvil,

y a la tierra movible circulando,

siguiendo por la eclíptica el buen orden,

que los signos observan cada un año,

con otro movimiento, que se cumple

del día natural en el espacio:

con tal arte descifra las estrellas,

que se obstenta a la vista sobrehumano.

En un tomo escribió la Astronomía,

dividiendo en seis libros celebrados:

Dejó tablas de fijas y de errantes, aunque cuesta entenderlas gran trabajo ${ }^{30}$.

28 Gonzalo Antonio SerRano, Astronomía universal, teórica y práctica..., Córdoba, en la Imprenta del autor..., por Pedro Arias de la Vega y Domingo Acosta, 1735.

29 SERrano, Astronomía universal, teórica y práctica, pág. XXIX.

30 Riccioli, Tablas philipicas catholicas o generales de los movimientos celestes, pág. [19]. 
3) y tanto en el poema como en la guía aparece Tycho Brahe de forma protagonista; la guía le dedica más de dos pliegos, solo igualado por el propio Gonzalo Antonio Serrano, mientras que los demás autores apenas cuentan con un párrafo. Ello puede deberse a que la teoría de Tycho Brahe se acercaba a la propuesta por Copérnico, pero mantenía a la tierra fija respecto al universo, de forma que esta fue la teoría a la que Iglesia se acercó tras la de Ptolomeo (teoría geocéntrica), descartando, en un primer momento, la teoría heliocéntrica.

Teniendo esta mirada presente, vemos lo que sería para los científicos del momento el origen de la historia científica a través del romance endecasílabo. Algunos de los rasgos del poema nos permiten considerarlo como característico de todo un conjunto de composiciones que reflejan la convivencia de la ciencia y la poesía, tal como hemos apreciado en las diferentes calas bibliográficas, aunque la falta de espacio no nos permita un mayor detalle de cada una de ellas.

La voluntad de dar relieve a la materia y encajar un nuevo léxico conduce a la elevación del discurso. La primera referencia es el uso del romance heroico, ya empleado en el Alto Barroco, aunque aquí adquiere unas características específicas a partir de las posibilidades de su extensión, propicia lo discursivo, y de la dignidad estética del abandono del arte menor. La elección del endecasílabo acerca al poema a algunos de los rasgos de la épica pero conciliándolos con el directo carácter didáctico ${ }^{31}$.

El poema comienza con el ritual introductorio que se repite en la épica culta, con una petición de inspiración al oráculo de Delfos. Este comienzo es más acorde a un poeta que a un científico, cómo es el caso, ya que el desarrollo de la ciencia se puede considerar opuesto a la necesidad de inspiración.

Como hemos comentado, en esta historia de la ciencia astronómica, sus orígenes comienzan en Dios, como el principio de todo:

$$
\begin{aligned}
& \text { Aónico prodigio, dulce acento, } \\
& \text { oráculo de Delfos soberano, } \\
& \text { inspirad a mi numen infecundo, } \\
& \text { romped de mi rudeza los candados. } \\
& \text { A cantar los progresos de la Ciencia }
\end{aligned}
$$

31 Otro ejemplo del uso del arte mayor con fines didácticos lo encontramos en Fernando CaLvo, Libro de Albeiteria en el qval se trata del cavallo, mvlo, y ijumento, y de svs miembros, y... enfermedades... Y vltimamente, se ponen mvchas, y svtiles questiones, y Preguntas, con sus Respuestas... y vn nuevo Arte de herrar en Octavas... Compvesto por - Madrid, Por Andrés Garcia y Lucas Antonio de Bedmar; A costa de Iuan de San Vicente, mercader de Libros. Vendese en su casa en frente de San Felipe, 1671. Introduciendo las octavas que menciona el título leemos: «Dialogo del arte de herrar entre el maestro Fernando Caluo, y el discipulo de este arte [...] el qual el Arte de Herrar và en octauas, compuestas por el mismo Autor; porque con mas facilidad le pueda el discipulo encomendar a la memoria...», fol. 353. 
Física, no frustránea de los astros

se dirige mi Musa, haciendo origen

de mi cómputo al Padre Protoplasto ${ }^{32}$.

Vemos en este fragmento cómo aún aparecen elementos propios del Barroco, como la figura retórica del hipérbaton (versos 5-7) o unos términos recurrentes en el ritual introductorio culto como inspiración y musa.

En el poema encontramos alusiones a divinidades mitológicas. Concretamente, en el texto expuesto la podemos hallar en el primer verso mediante la figura retórica de la perífrasis. Entrando en el léxico, observamos cómo hay abundante uso de cultismos (numen, infecundo, frustránea...), al igual que un vocabulario más especializado y científico como ciencia, física y protoplasto, aunque sin ir más allá de una mera apariencia de innovación que queda totalmente sobrepasada por los moldes barrocos en el poema. Incluso el propio término protoplasto fue ya usado en 1612 por Lope en la obra Pastores de Belén, prosas y versos divinos ${ }^{33}$.

\section{Ciencias aplicadas}

Acudiendo ahora a las obras de Ciencias aplicadas vemos que en la BDH hay 4.974 obras digitalizadas. Tras aplicar los filtros antes mencionados, tenemos un resultado de 65 obras afines a nuestros criterios.

Bajo el subgrupo de ciencias aplicadas destacan los libros de las ciencias médicas. La medicina ha jugado un papel fundamental dentro del movimiento novator y en el desarrollo de las que fueron las nuevas bases científicas. Entre los siglos XVII y XVIII la medicina, tanto en su vertiente práctica como conceptual, cambió contraponiendo los nuevos avances a las estructuras médicas del Antiguo Régimen.

Es sabido que la época de impresión de estas obras estuvo marcada por la polémica que enfrentó a la medicina más conservadora y la medicina moderna. Los especialistas en esta controversia médica lo acotan entre:

[...] casi cuarenta años, los que median entre dos sonoras controversias que jalonan la irrupción de la medicina moderna en España, por utilizar la periodización tradicional de López Piñero y Peset Llorca, inteligible por todos: la generada

32 Riccioli, Tablas philipicas catholicas o generales de los movimientos celestes, pág. [13], vv. 1-8.

33 Félix Lope de Vega, Pastores de Belén, prosas y versos divinos, Madrid, Por Ioan de la Cuesta, vendese en casa de Alonso Perez, mercader de libros, 1612, pág. 192. 
en torno a la Carta de Cabriada, publicada en 1687, y la que se inició con la aparición del primer tomo del Teatro crítico universal, de Feijoo, en $1726^{34}$.

En nuestro corpus encontramos autores médicos de una relevancia crucial en esta polémica, como los citados Juan de Cabriada, Muñoz y Peralta, además de Zapata y Juanini, entre otros. En las obras impresas estudiadas de estos médicos (y otros más) aparecen poesías que, como veremos, están íntimamente relacionadas con el propio papel que jugaban sus obras.

El conflicto entre médicos surgió:

[...] entre los llamados médicos revalidados de Sevilla —aquellos que abandonaban la Universidad con el grado de bachiller en medicina, se dedicaban a practicar con un médico durante un par de años y se revalidaban ante el Protomedicato para poder ejercer-y los médicos doctores del claustro sevillano, quienes además de la reválida poseían el grado universitario de doctor ${ }^{35}$.

A través de la poesía también se sancionaba esta disputa, como sucede en la obra El monstrvo horrible de Grecia mortal inimigo del hombre... ${ }^{36}$ de Gonzalo Bustos de Olmedilla. El autor, ajeno al movimiento novator, pero fuerte activista contra los galenistas y sus sangrías, imprime junto a su texto un romance paratextual compuesto por el doctor Jacinto de la Fuente Ocon y Lumbreras titulado Del mismo Author (Fuente Ocon) a los Barberos:

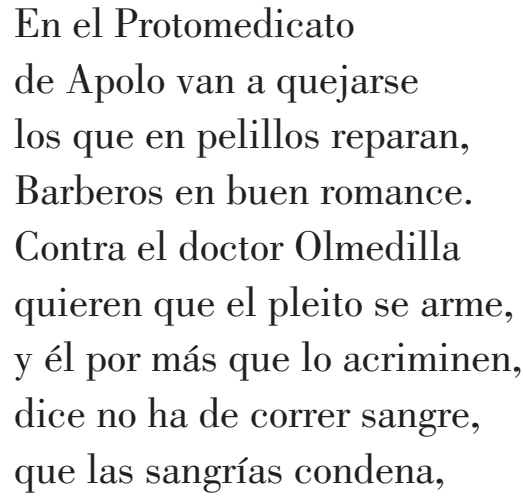

34 José Pardo Tomás, Àlvar Martínez Vidal, «Las consultas y juntas de médicos como escenarios de controversia científica y práctica médica en la época de los novatores (1687-1725)», Dynamis. Acta Hispanica ad Medicinae Scientiarumque Historiam Illustrandam, 22 (2002), pág. 304.

35 Pardo Tomás, Martínez Vidal, «Las consultas y juntas de médicos como escenarios de controversia científica y práctica médica en la época de los novatores (1687-1725)», pág. 305.

36 Gonzalo Bustos de Olmedilla, El monstrvo horrible de Grecia mortal inimigo del hombre..., Lisboa, en la officina de Iuan de la Costa, 1675. 
es el cargo que le hacen, en daño de los Barberos, que otro agujero no saben.

Lo que se usa no se escusa, y en aqueste mundo frágil, barberos y pretendientes de pies y brazos se valen. Que son los gastos terribles, y que para sustentarse, si no sacan de las venas, no tienen de qué lo saquen ${ }^{37}$.

El ámbito de discusión de ambos grupos se centraba en la primacía de uno sobre el otro a la hora de realizar las consultas y las juntas de médicos. Tras el problema protocolario, como nos indican Pardo y Martínez,

[...] se hallaba una cuestión de vital importancia, puesto que el orden en las juntas jerarquizaba públicamente a los médicos, les confería una distinción tanto en el seno de la profesión como, sobre todo, ante el «público» que constituía su clientela y, por tanto, su medio esencial de vida, de prestigio y, en última instancia, de nivel económico ${ }^{38}$.

Nos encontramos ante una problemática similar al del posicionamiento de campo de poder que hemos analizado en la poesía aurisecular y posterior ${ }^{39}$. Como venimos estudiando desde el grupo de investigación PAso (Poesía Andaluza del Siglo de Oro) vemos, salvando las distancias entre ambos objetos de estudio, que uno de los recursos de posicionamiento dentro de este campo social es el uso de la poesía paratextual, la más frecuente en los libros científicos del momento. La mayor parte de la controversia médica ha discurrido

37 Bustos de Olmedilla, El monstrvo horrible de Grecia, págs. 36-37.

38 Pardo Tomás, Martínez Vidal, «Las consultas y juntas de médicos como escenarios de controversia científica y práctica médica en la época de los novatores (1687-1725)», pág. 305.

39 Al respecto cito la obra de Ignacio García Aguilar (ed.), Tras el canon. La poesía del Barroco tardío, Editorial Academia del Hispanismo, Vigo, 2009. También está la labor desarrollada por el grupo de investigación PASO (Poesía Andaluza del Siglo de Oro), en concreto en Begoña LóPEz BuEno (ed.), El canon poético en el siglo XVI (VIII Encuentros Internacionales sobre Poesía del Siglo de Oro), Sevilla, Secretariado de Publicaciones de la Universidad / Grupo PASO, 2008; Begoña LóPEz Bueno (ed.), El canon poético en el siglo XVII (IX Encuentros Internacionales sobre Poesía del Siglo de Oro), Sevilla, Secretariado de Publicaciones de la Universidad / Grupo PASO, 2010. 
por derroteros manuscritos, principalmente epistolares, pero ha quedado un remanente importante en letras de molde con el que poder trabajar. Es más, aunque la cantidad de dichas obras impresas respecto a las manuscritas sea muy inferior, el rango de alcance de las primeras excede con creces a la circulación manuscrita.

Gran parte de las obras poéticas impresas de los siglos XVI y XVII incluían versos paratextuales, composiciones poéticas situadas entre (o en) el prólogo, licencia o censura, pero, sobre todo, hallamos poemas laudatorios ${ }^{40}$. Es un uso editorial asentado en la época, síntoma de una práctica que se enmarca dentro de la sociología de la edición de un período que no entiende de materia, sino de finalidades sociales. Estas eran diversas y lo mostraremos con algunos ejemplos que hallamos en las obras científicas seleccionadas ${ }^{41}$ :

a) alabanza y ensalzamiento del autor de la obra a estratos intelectuales superiores, en la «Décima de José Antonio de Eguizaval» ${ }^{42}$ :

De Filosofía la vena
preservativa al veneno
descubres, que el gran Galeno
no conoció, ni Avicena:
Mayor tu fama resuena,
Don Tomás, pues les das ley,
por ciencia, estado y por Grey;
y así es justo apellidallos
tus cautivos, sus vasallos,
y a ti coronarte Rey.

b) mecenazgo, en la «Dedicatoria al mecenas (Don Pedro Ceballos, Mariscal de Campo de los Reales Ejércitos de su Majestad)» ${ }^{43}$ :

40 Obra de obligada consulta al respecto es Ignacio García Aguilar, Poesía y edición en el Siglo de Oro, Madrid, Calambur, 2009.

${ }_{41}$ Ya hemos incidido sobre esto en las obras de ciencias puras, ya que es una práctica asentada editorialmente. Mostramos ejemplos de esta mecánica en las obras de medicina, pero los resultados podrían ser extrapolables a cualquiera de las demás subcategorías.

42 Tomás Murillo y Velarde, Nveva, y varia decision, Ivridica, y medica en que se trata, si se pvede por los cadaveres conocer si han mverto, o no de venenos, y preservacion y cvracion dellos... sv avtor —, [Madrid?], [s. n.], [1675], pág. 16. Según la BNE la edición consta de 5 emisiones conocidas en las que cambia la persona a la que va dirigida y el escudo de armas.

43 Félix Fermín Eguía y Harrieta, Escrito abstracto de los mejores autores, de las virtudes, y para què enfermedades son utiles, y de sus ingredientes, las Aguas Minerales de Trillo, del Molàr, de Arnedillo, de Sacedòn, y de Buen-dia, que son las que regularmente practican los Cortesanos de esta Corte de Madrid, para 
Otros, aunque pocos, podrán

en una u otra materia,

mostrarse y decir son sabios:

Por en las dos a un tiempo,

sobresaliendo en su regla,

solo se ve en los Ceballos.

c) ofensiva frente algún enemigo, en la «Décima de Fr. Antonio de San Guilherme, Prior del Convento de Graça de la Villa de Loyle» ${ }^{44}$

Roca convence a Ribera

con locuciones Reales,

y apurando a sus cristales,

cristal de Roca se esmera,

Ribera es cuerpo en carrera:

Roca el alma, a quien bien toca

decir verdad en su boca:

Ya la Ribera presume

correr de cisne con pluma,

volando al pie de tal Roca.

d) defensa de la doctrina propuesta en la obra, en el soneto acróstico «Escrito y sentido por F. J. D. M.» ${ }^{45}$ :

D.e este acertado escrito los acentos

Juzga mi musa con afecto llano

Un asombro, un portento y un ufano

Atlante, y de Memnon vivos alientos.

Nadie ignora los únicos portentos

De la Quina, que explica aquesta mano,

Esgrimiendo ligero, fuerte, ufano

libertarse de sus respectivas dolencias, y lo que deben executar antes, en el baño, y despues de èl... añadida la noticia de las aguas de Humera nuevamente descubiertas. Por el doctor —, [Madrid], Se hallarà... en casa de Diego Barthelemi... Puerta del Sol..., 1745, pág. 7.

44 Antonio de Monrava y Roca, A un mismo tiempo Feijoo defendido y Ribera convencido, en abatimiento de la Medicina de Hipocrates, y Galeno Para desengaño de los no-pocos entendidos, Españoles, y Portugueses... su autor —, Antuerpia, En la Officina Plantiniana, 1732, pág. 29.

45 Juan Muñoz y Peralta, Escrvtinio Phisico Medico de vn peregrino especifico de las calentvras intermitentes, y otros achaques, motivado de vn libro qve escrivia el Dr. D. Joseph Colmenero... por Don —, Sevilla, Por Jvan de la Pverta. En las Siete Rebuelas, 1699, pág. 24. 
Periodos de invictos fundamentos.

Es el asumpto digno de tal pluma,

Reglada con acierto, e inmortal gloria

A la más fija, y firme experiencia;

La claridad alabo, y breve suma,

Tocando, y publicando la victoria,

¡Admírese la Atenas de tal Ciencia!

Pero no solo en la poesía preliminar hemos visto el uso que de ella hacían los médicos del siglo XVIII. La obra Residencia medico-christiana contra el theatro critico vniversal... ${ }^{46}$ en que el doctor don Bernardo López de Araujo y Ascarraga, médico de los Reales Hospitales, acomete contra la obra de Benito Feijoo, se cierra con esta décima:
Treinta y ocho papas dados, y cardenales doscientos, quince mil y setecientos santos ya canonizados. Siete mil arzobispados, obispados quince mil, doctores diez y seis mil tienes, Santa Religión, de Benito igran blasón! De estos San Benitos mil ${ }^{47}$.

Y continúa López de Araujo:

Tiempos ha que se escriviò esta Dezima, aunque puede ser que V. Rma. no aya tenido de ella noticia: natural cosa es que aya crecido en su Sagrada Religion el numero de Santos Doctores, y Prelados casi en infinito hasta oy: lastima será, que el precioso talento de V. Rma. no se dedique a acrecentar el numero. FINIS.

El objetivo de la décima era cerrar, apuntillar una crítica construida desde una argumentación médico-religiosa frente al Teatro Crítico Universal publicado un año antes. Es otro apunte del uso de los versos en la ciencia del siglo XVIII,

46 Bernardo López de Araujo y AscárRaga, Residencia medico-christiana contra el Theatro Critico Vniversal, en honor de la medicina, lustre de sus Professores, y desengaño del Vulgo... Compvesta por —, Madrid, [s. n.], 1727.

47 López de Araujo y Ascárraga, Residencia medico-christiana, pág. 124. 
ya que como hemos dicho, estas polémicas agudizan el ingenio de los autores y nutren a la disciplina de verdaderos manuales teóricos científicos.

Otro ejemplo que sigue la misma línea lo hallamos en la obra Satisfaccion publica, a una poco secreta calumnia, sobre la quasi universal constitución pleuritico catharral del año $1716^{48}$, escrita por el bachiller D. Francisco Manuel de Herrera Carrasco y publicada a razón del escrito latino de Bernabé Rodríguez de Texada ${ }^{49}$. Como colofón de la satisfacción de Herrera leemos la siguiente redondilla que dictó la razón a un amigo del autor:

$$
\begin{aligned}
& \text { Argumentos de Manuel, } \\
& \text { hoy convencen a Texada, } \\
& \text { pues este no prueba nada, } \\
& \text { y su intento prueba aquel }{ }^{50} \text {. }
\end{aligned}
$$

Aparte del mero reproche, haremos alusión a dos aspectos relevantes. El primero es que, en el encabezado del poema, quien dicta la pluma del amigo del autor es la razón (con todo lo que el término acarrea ya en el primer tercio del siglo XVIII). El segundo es el énfasis que el autor pone en su poema acerca de que para tener la razón, a través de los argumentos, hay que basarse en pruebas, en la experimentación científica y su posterior verificación. Para reafirmar mis palabras apelo a la primera décima que sigue a la redondilla:

$$
\begin{aligned}
& \text { Es la razón natural, } \\
& \text { tan convincente razón, } \\
& \text { que no hay contraria opinión, } \\
& \text { que pueda ponerla en mal. } \\
& \text { En esta cuestión casual, } \\
& \text { Herrera toma el nivel, } \\
& \text { y de su doctrina fiel, }
\end{aligned}
$$

48 Francisco Manuel de Herrera Carrasca, Satisfaccion publica a una poco secreta calumnia sobre la quasi universal constitucion pleuritico catharral del año 1716..., Valladolid, En la Imprenta de la Real Chancilleria, que es de la Viuda de Joseph de Rueda, vive à la Calle de Samano, 1717.

49 La descripción completa de la situación fue: «Don Francisco Manuel de Herrera Carrasco, graduado en Salamanca, y médico de Aguilar del Campo, firmó en dicho pueblo á 3 de Junio de 1716 una consulta sobre la curación del caso; á la qual contextó el doctor Bernabé Rodríguez de Texada, con una impugnación latina, que firmó en Burgos á 15 de Octubre del mismo año: resentido de ello el expresado Herrera, satisfizo con otra que tituló: Satisfaccion pública...» y se puede encontrar y ampliar la noticia en Joaquín VILLALBA, Epidemiologia española, ó historia cronológica de las pestes, contagios, epidemias y epizootias que han acaecido en España desde la venida de los cartagineses hasta el año 1801... Tomo I, Madrid, en la Imprenta de D. Fermín Villalpando, 1803, pág. 98.

50 Herrera Carrasca, Satisfaccion publica a una poco secreta calumnia, pág. [107]. 
hace espada para herir,

pues no es fácil resistir

argumentos de Manuel $^{51}$.

Observamos que es en estas obras donde el papel de la poesía adquiere una mayor incidencia pragmática.

Como última incursión nos detendremos en dos obras de otra subcategoría, como es la albeitería. En esta disciplina también sucede lo mismo que aconteció en la medicina: las rencillas profesionales e intelectuales se dirimen a través de la poesía en sus obras impresas. En 1732 se publicó la obra Coloquios de Albeyteria, que tuvieron, sobre la cura de la Lupia Tumorossa, que diò à luz Francisco Garcia Cabero... ${ }^{52}$ compuesta por Francisco Benavides, Antonio Guzmán y Lázaro Cortázar, quienes, según reza en la portada de la obra, eran «mancebos de Herrador, y asistentes en esta Corte de Madrid». En el prólogo de este coloquio, que sale a la luz con el objeto de enmendar la plana a su colega García Cavero, leemos cuatro décimas atacando a otro autor, preludiadas «y ansi, parece que viene bien aqui aquella Decima, que se dixo, no sè si al mismo intento, por un Mozo del camino, que es como se sigue»:

Si acaso tu presumpcion
te inclina a ser imprudente,
advierte, que lo elocuente
no está en la imaginación,
sino en modestia, y razón:
Y si es que tu pluma avara,
con reflexión lo repara,
conocerá su desvelo,
que ninguno escupe a el cielo,
que no le caiga en la cara ${ }^{53}$.

Las otras tres décimas que le siguen se mantienen al mismo nivel y con el mismo ímpetu acusador.

A esta obra, y en el mismo año, la siguió una nueva respuesta en que Francisco García Cavero responde a todas las cuestiones dialogadas de la obra de

51 Herrera Carrasca, Satisfaccion publica a una poco secreta calumnia, pág. [107].

52 Francisco Benavides et al., Coloquios de Albeyteria, que tuvieron, sobre la cura de la Lupia Tumorossa, que diò à luz Francisco Garcia Cabero y escrivieron —: Dividenlos en tres partes: El primero, sobre la introduccion; el segundo, sobre su difininicion [sic]...; y el tercero, sobre su curacion., Madrid, [s. n.], 1732.

53 Benavides et al., Coloquios de Albeyteria, pág. 11. 
los tres mancebos de albeitería y muestra en concreto, y es la parte que más nos interesa ahora, cómo son recibidos y contestados los versos acusadores:

[...] mire que traza de presentarse estos [los mancebos] a lidiar en el Campo Literario, quando no saben, ni se espera, que sepan formar vna Clausula.

[...] Cerca del fin del Prologo ay una Decima, que dice ser hecha por un Mozo del Camino; y aunque es cierto esta sin las reglas Poeticas, la dexo para quando llegue el caso.

Otra aparece à la buelta, que es muy parecida à la primera, y el primer verso se parece a aquellos con que empiezan los Romances, que hacen los Ciegos, de la vida, prision, y muerte algun Facinoroso: pues dice ansi: Detenga el buelo tu pluma. Y cierto, que si en lugar del $t u$, huviera puesto su Autor el $m i$, fuera gran cosa, y en esse caso dixera: Detenga el buelo mi pluma, que no sabe lo que hace.

Siguese otra, que desde la nota hasta el fin de ella, està hecha con poquisima atencion... ${ }^{54}$

García Cavero continúa comentando las poesías paratextuales en los siguientes folios, pero con el extracto aquí mostrado observamos cómo para el veterinario es tan importante resarcirse de los versos calumniosos como contestar a la propia teoría científica contraria a la suya.

\section{Últimas reflexiones}

Llegados a este punto, es hora de reflexionar sobre las cuestiones que al comienzo del artículo planteábamos. Todas las composiciones poéticas, o al menos la inmensa mayoría, tienen un máximo común denominador, unos rasgos compartidos que merecen ser destacados.

Las funciones de los versos en los tratados científicos estudiados son, principalmente, dos: paratextuales, con una justificación externa, e intratextuales, que sirven de refuerzo de efecto de la materia tratada.

La mayoría de las poesías paratextuales son composiciones poéticas en alabanza del autor o de su obra cuando no de ambas, que con frecuencia actuaban como acicate entre disputas profesionales. Eran pesos que hacían decantar la balanza hacia algunos de los lados cuando se dirimían posturas personales y

54 Francisco García Cavero, Adiccion racional, y methodica, a la curacion de la lupia tvmorosa, y destierrro de ignorancias, hijas de los errados conceptos de Joseph Andres Moraleda, maestro Herrador... su autor —, Madrid, [s. n.], 1732, págs. 48-49. 
teóricas diferentes. La imposición de unas ideas o teorías científicas sobre otras no era baladí y tenían consecuencias en la vida de los que la promulgaban, bien para mejorar su estatus o bien para perderlo si su propuesta no era la elegida.

Las poesías intratextuales actúan como una ampliación de los niveles de recepción, en algunos casos facilitando la comprensión del texto al que acompañan. No volveremos a mencionar la importancia en la didáctica y aprendizaje de los conocimientos a través de los versos, pero sí cómo estos eran utilizados como juego mnemotécnico por los lectores y, en muchos casos, por aprendices y discípulos.

Llegamos a la conclusión (aproximativa) de que elementos poéticos formales barrocos se han mantenido, tanto en métrica como en retórica. Para los moldes epigramáticos se reservan principalmente composiciones endecasílabas y octosílabas. En cambio, para los poemas más narrativos y expositivos se utiliza el romance, por los motivos que antes hemos expuesto acerca de su extensión y su capacidad de albergar diferentes conceptos e ideas.

Otro elemento común es la contaminación léxica que se da en los versos. Estos tienen una gran carga científica que ayuda a la ampliación del concepto y lleva a la poesía más allá de la sentimentalidad lírica. Buscan en el lector una constante sensación de novedad y, en ciertos casos, de elitismo científico destinado a lectores especializados, lo que aleja a su vez a los neófitos de las técnicas puras y aplicadas.

Los agentes de estos versos son personas afines al autor. Remarcamos cómo la inmensa mayoría de estas composiciones están realizadas por familiares y/o discípulos directos de los autores de las obras científicas. Siguiendo la clasificación de poetas propuesta por Pedro Ruiz ${ }^{55}$, la mayoría de nuestros protagonistas se pueden agrupar bajo los títulos de letrados, que vienen a completar la función canonizadora [normalmente] en preliminares, y amateurs, que no siempre deben ser nobles ni personas alejadas de las imprentas. En este sentido, juegan un papel importante las academias científicas. En multitud de casos, las poesías están compuestas por miembros de una misma academia. Un estudio en profundidad sobre este hecho podría constatar que en las reuniones de estos (primeros) científicos, la poesía tendría cabida de un modo similar al que tenía en las academias literarias que tanto proliferaron en el siglo XVIII.

En estas composiciones encontramos un espacio de intersección, un punto de conexión entre las diferentes materias poéticas. Por un lado, esta poesía no es un mero adorno en las obras científicas que hemos visto, cumple unas funciones determinantes que posicionan social y profesionalmente a los autores. Por

55 RuIz Pérez, «Para la historia y la crítica de un período oscuro: la poesía del bajo barroco», pág. 20. 
otro, observamos un caso en que sobre una base científica se plasman inquietudes religiosas. Esta es una de las particularidades que se dio en la sociedad de mediados del siglo XVIII, donde la luz y la razón se van abriendo paso y se enfrentan con una profunda religiosidad que no queda desterrada de la sociedad, sino que coexisten desde una perspectiva más inquisitiva, ya superado el desengaño barroco aferrándose a la ciencia y a la naturaleza. Un ejemplo lo tendríamos en el romance endecasílabo analizado (Progresso de la Astronomía...) que desprende un fuerte sentimiento religioso (hacemos hincapié en la fecha de publicación, 1745), en el que se entremezcla ficción y realidad, biblia y ciencia. Hay una clara apuesta por la concepción cristiana del universo por parte de la poesía científica (reflejo al menos de la ciencia cordobesa de la que procede el romance). Evidentemente, y con inquisiciones por medio, no sería normal una apuesta radical en su contra.

Pensamos que esta poesía no va acorde con el contenido científico de los libros del corpus, libros de autores innovadores que, dentro de las restricciones sociales e ideológicas, van aportando avances y mentalidad europea.

Queda patente una persistencia y extensión de unas prácticas editoriales asentadas en las centurias anteriores. Versos que acompañan a la prosa, que la matizan, completan y adornan. Pero, a pesar de todo, el papel que jugaban estos versos era secundario, más como la perpetuación de una tradición editorial asimilada por el público lector que como elementos imprescindibles para la compresión del conocimiento que pretendían transmitir. Pero esto denotaba a su vez una alta capacidad de los hombres de ciencia en el entendimiento del entretenimiento de las musas y su arrojo a la hora de publicar sus poemas. Lo mismo pensamos si nos centramos en la recepción de estos tratados y su público objetivo.

Al principio del trabajo hacíamos referencia al pensamiento de François Lopez sobre la capa impermeable que aislaba España en la era de los novatores, que a la postre no fue tan efectiva, y sí se puede constatar que no éramos ajenos del todo a los avances científicos europeos, aunque estos se manifestaban más en los tratados científicos que en la poesía que contenían.

Esperamos haber arrojado algo de luz sobre estos versos científicos contenidos en las obras destinadas a iluminar el camino de los españoles hacia la Ilustración. 